Special issue of the International Conference on Computational and Experimental Science and Engineering (ICCESEN 2014)

\title{
Optimization of Circular Antenna Arrays Using a Differential Search Algorithm
}

A. RECIOUI*

IGEE, University of Boumerdes, Institute of Electrical Engineering and Electronics, 35000 Boumerdes, Algeria

Circular and concentric circular antenna array patterns are synthesized to generate array factors with minimum side-lobe level and high directivity. The comprehensive learning differential search algorithm is used for synthesizing a one-and a three-ring circular and concentric circular antenna array with thirty elements. This synthesis is done by finding the optimum inter-element spacing of rings, phases and /or positions that give optimum side lobe level. The computational results show that side lobe level is reduced significantly in non-uniform case for concentric in comparison with a single ring.

DOI: 10.12693/APhysPolA.128.B-7

PACS: 84.40.Ba, 52.40.Fd

\section{Introduction}

Antenna arrays have been widely used in different applications [1]. Among the different types of antenna arrays, circular arrays have become more popular in mobile and wireless communications [2-5]. The circular array, in which the radiating elements are placed on circular rings, is an array of very great practical interest. These applications are multiple: radars, sonar, terrestrial and space navigation and much of other systems [6].

Antenna arrays possess two properties that are very important performance criteria: side-lobe level (SLL) and the directivity. Directivity is a measure of how the array can accentuate the radiated power in a particular (usually a desired) orientation. Side-lobe level accounts for how much interference can the array gather besides the desired signal. These two properties turn out, however, not to go hand in hand in the sense that optimizing for one of them affects the other. Hence, a compromise has to be considered by the design engineer while trying to optimize the array performance in terms of these two characteristics $[7,8]$.

Circular shaped antenna arrays have been optimized previously in literature for different purposes [9-11]. A first metaheuristic approach towards the design of circular arrays is found in [12] where a real-coded genetic algorithm (GA) has been applied for designing circular arrays with maximal side lobe level reduction coupled with the constraint of a fixed beam width. Shihab et al. in [13] applied the particle swarm optimization (PSO) algorithm to the same problem and achieved better results as compared to those reported in [12]. Recently, Panduro et al. [14] compared three powerful populationbased optimization algorithms - PSO, GA, and differential evolution (DE) on the design problem of scanned circular arrays.

*corresponding author; e-mail: recmad2006@yahoo.fr
In this work, an attempt to apply a differential search algorithm (DSA) to the design of a directive and low sidelobe levels non-uniform circular antenna arrays. Both simple circular arrays and multi-ring concentric arrays are considered.

\section{Problem formulation}

The array factor of circular antenna arrays is given as [1]:

$$
A F(\theta, \phi)=\sum_{n=1}^{N} I_{n} \exp \left(\mathrm{j}\left(k a \sin (\theta) \cos \left(\phi-\phi_{n}\right)+\alpha_{n}\right)\right),(1)
$$

where $I$ is the excitation amplitude of element $n, \phi_{n}$ is its position and $\alpha_{n}$ is the excitation phase. $k$ is the wave number and $a$ is the circle radius.

For the concentric circular array with $M$ rings and $N_{m}$ elements in the corresponding $m$-th ring, the array factor is given as

$$
\begin{aligned}
& A F(\theta, \phi)= \\
& \quad \sum_{m=1}^{M} \sum_{n=1}^{N_{M}} I_{n m} \exp \left(\mathrm{j} k a_{m} \sin (\theta) \cos \left(\phi-\phi_{n m}\right)+\alpha_{n m}\right) .
\end{aligned}
$$

The objective function to be minimized is taken to be merely the side-lobe level and the directivity of the array is considered as another performance parameter to compare with while evaluating the end result.

\section{The differential search algorithm}

The differential search algorithm (DSA) models swarm behaviour. Imagine a swarm of bees looking for the most fertile feeding location in a field. Each bee has a location in the three-dimensional space and it evaluates every position for the absolute fitness [15]. This fitness will, for this example, be a positive number which increases with increasing fertility (or simply the density of flowers). The bee remembers the spot where it encountered the best fitness and also shares this information with the other bees, so that the entire swarm will know the global best position. 


\section{Results and discussions}

\subsection{Single ring circular array optimization}

Table I summarizes the results of single ring circular array opimization.

TABLE I

Summary of the obtained results for a single ring circular array.

\begin{tabular}{c|c|c|c|c}
\hline \hline type of variation & SLL & directivity & $\begin{array}{c}\text { ratio } \\
\text { dir./SLL }\end{array}$ & $\begin{array}{c}\% \text { decrease } \\
\text { to uniform } \\
\text { case }\end{array}$ \\
\hline amplitude-only & -11.52 & 26.39 & 2.2906 & $46.3 \%$ \\
position only & -9.07 & 20.2713 & 2.3232 & $45.54 \%$ \\
phase-only & -13.01 & 32.1879 & 2.4733 & $42.02 \%$ \\
amplitude-phase & -13.24 & 28.0895 & 2.2110 & $48.17 \%$ \\
amplitude-position & -10.94 & 24.5409 & 2.2432 & $47.41 \%$ \\
amplitude-phase- & -12.57 & 26.8419 & 2.1346 & $49.96 \%$ \\
position & & & &
\end{tabular}

The uniform case does not exhibit the best directivity/side-lobe level compromise. Indeed, the ratio directivity/side-lobe level in all non-uniform cases is better than the uniform one. Hence, the non-uniform cases present themselves to be a better choice regarding these two performance measures. Side-lobe levels were generated by the amplitude-phase and phase-only variations i.e. $\mathrm{SLL}=-13.2433 \mathrm{~dB}$ and $\mathrm{SLL}=-13.0100 \mathrm{~dB}$, respectively, with corresponding highest directivities i.e. $\mathrm{DIR}=32.1879 \mathrm{~dB}$ and $\mathrm{DIR}=28.0980 \mathrm{~dB}$, respectively.

\subsection{Concentric circular array optimization}

Table II presents the results of concentric circular array optimization.

TABLE II

Summary of the obtained results for a multiple ring concentric circular array.

\begin{tabular}{c|c|c|c|c}
\hline \hline type of variation & SLL & directivity & $\begin{array}{c}\text { ratio } \\
\text { dir./SLL }\end{array}$ & $\begin{array}{c}\% \text { decrease } \\
\text { to uniform } \\
\text { case }\end{array}$ \\
\hline amplitude-only & -22.23 & 30.5181 & 1.3728 & $52.96 \%$ \\
position only & -15.09 & 33.9794 & 2.2516 & $25.53 \%$ \\
phase-only & -21.12 & 24.6510 & 1.1668 & $59.85 \%$ \\
amplitude-phase & -21.24 & 53.0441 & 2.4963 & $14.11 \%$ \\
amplitude-position & -17.86 & 35.4347 & 1.9840 & $31.74 \%$ \\
amplitude-phase- & -18.37 & 31.9612 & 1.7891 & $38.44 \%$ \\
position & & & &
\end{tabular}

Once again, it is noticed that the non-uniform cases produce a better compromise between the array directivity and its side-lobe level. Indeed, the ratio directivity/side-lobe level is not the best compromise and hence the adoption of this situation is not beneficial in terms of the two performance criteria. Side-lobe levels were generated by the cases of amplitude-only, phaseonly and amplitude-phase variations. Compared to the single-ring circular array, better values are noticed which favors the deployment of the concentric circular arrays over single-ring arrays.

\section{Conclusions}

In this work, a recently developed ecologically inspired meta-heuristic algorithm called differential search algorithm has been used to optimize both circular and concentric arrays. From the obtained results we observe that the side-lobe levels obtained from non-uniform cases are better compared to those of uniform antenna arrays. Furthermore for non-uniform case, better results have been observed for concentric antenna arrays in comparison with just a single ring.

\section{References}

[1] C.A. Balanis, Antenna Theory: Analysis and Design, 3rd ed., Wiley, New York 2005.

[2] S. Zainud-Deen, E. Mady, K. Awadalla, H. Hars-Her, in: IEEE Antennas Propagation Symp., IEEE, Albuquerque 2006, p. 3399

[3] L. Pesik, D. Paul, C. Railton, G. Hilton, M. Beach, Electron. Lett. 42, 787 (2006).

[4] M. Panduro, A. Mendez, R. Dominguez, G. Romero, J. Electron. Commun. 60, 713 (2006).

[5] W. Mahler, F. Landstorfer, in: IEEE/ACES, Int. Conf. on Wireless Communications and Applied Computational Electromagnetics, 2005, p. 1006.

[6] R.J. Mailloux, Phased Array Antenna Handbook, Artech House, 1994.

[7] A. Recioui, Microw. Opt. Technol. Lett. 49, 1619 (2007).

[8] A. Recioui, A. Azrar, H. Bentarzi, M. Dehmas, M. Chalal, Int. J. Microw. Opt. Technol. 3, 524 (2008).

[9] M.I.H. Dessouky, A. Sharshar, Y.A. Albagory, Prog. Electromagn. Res. PIER 65, 187 (2006).

[10] L. Gurel, O. Ergul, Prog. Electromagn. Res. PIER 85, 243 (2008).

[11] M. Dessouky, H. Sharshar, Y. Albagory, J. Electromagn. Waves Appl. 20, 2077 (2006).

[12] M. Panduro, A.L. Mendez, R. Dominguez, G. Romero, Int. J. Electron. Commun. (AEU) 60 , 713 (2006).

[13] M. Shihab, Y. Najjar, N. Dib, M. Khodier, J. Electric. Eng. 59, 216 (2008).

[14] M.A. Panduro, C.A. Brizuela, L.I. Balderas, D.A. Acosta, Prog. Electromagn. Res. B 13, 171 (2009).

[15] P. Civicioglu, Comput. Geosci. 46, 229 (2012). 